Revista de Psicología de la PUCP. Vol. XVII, 1, 1999.

\title{
LA EVALUACIÓN DEL DESARROLLO ${ }^{1}$
}

\author{
Jan J. F. ter $\mathrm{Laak}^{2}$ \\ Universidad de Utrecht
}

Se sostiene que la psicología del desarrollo es la "tercera disciplina de la psicología científica", enfatizándose el aspecto formal de la continuidad/cambio conductual. Los constructos que elaboran este aspecto son semánticos y no modelos matemáticos vacios o borrosos conceptos de sentido común. Se describe comprensivamente la evaluación como una matriz de cuatro componentes (teoría de los tests, constructos, tests, proceso diagnóstico) por tres niveles (sentido común, constructos científicos, modelos matemáticos). Se confrontan la psicología del desarrollo y la evaluación con la finalidad de captar y ampliar el significado de continuidad/cambio, como en la validación de constructos "convergentes" y "discriminantes". La teoría de los tests, los tests y el proceso diagnóstico ofrecen, respectivamente, modelos, instrumentos y un programa para evaluar la conducta.

Palabras claves: "Tercera" disciplina de la psicología científica, continuidad/cambio conductual, evaluación, teoría de los tests, constructos semánticos, tests, proceso diagnóstico.

\section{Assessment of Development}

It is argued that developmental psychology is the "third discipline of scientific psychology" , emphasizing the formal aspect of behavioral continuity/change. Constructs elaborating this aspect are semantical and not empry mathematical models or fuzzy common sense concepts. Assessment is comprehensively described as a matrix of four components (test theory, constructs, tests, diagnostical proces) by three levels (common sense, scientific constructs, mathematical models). Developmental psychology and assessment are confronted to conquer and extend the meaning of continuity/change, as in "convergent" and "discriminant" construct validation. Test theory, tests and the diagnostical proces respectively offer models, instruments and a program for assessing behavior.

Key words: "Third" discipline of scientific psychology, behavioral continuity/change, assessment, test theory, semantic constructs, tests, diagnosis.

1. Basado en una contribución al simposio Evaluación del Desarrollo en la Reunión Bienal de la Sociedad para la Investigación del Desarrollo del Niño, realizada en Washington, del 3 al 6 de abril de 1997.

2. Profesor asociado de la Universidad de Utrecht, Departamento de Psicología del Desarrollo. Heidelberglaan 2, 3508 TC Utrecht, Países Bajos. Correo electrónico: J.TERLAAK @FSW.RUU.NL. Fax: + 31302534777. 



\section{Psicología del desarrollo, la "tercera" disciplina de la psicología científica}

La psicología del desarrollo básicamente no es ni una disciplina experimental ocupada en los cambios en las variables dependientes como resultado de la manipulación de variables independientes; ni una disciplina correlacional ocupada en las covariaciones de las dimensiones sobre diferencias individuales. Dado que los humanos son seres históricos, sus conductas necesariamente están pautadas en el tiempo. Esta característica no está cubierta por las dos disciplinas y se precisa la perspectiva de la psicología del desarrollo.

La disciplina experimental presupone estabilidad después de la manipulación o neutraliza la variabilidad (p.e.estudios de modificación de conducta $n=1$ ) o corrige las tendencias en el tiempo (p.e. en los análisis de series temporales, ver Glass, Willson y Gottman, 1975). La disciplina correlacional presupone la estabilidad del patrón de covariaciones en el curso del tiempo.

Aunque la continuidad/cambio conductual parece obvio no están simplemente allí. Sólo pueden observarse, comprenderse y explicarse si están precedidos por constructos evolutivos y/o seguidos por interpretaciones evolutivas. Ejemplos son el cambio estructural (piagetano) del pensamiento pre-operacional al operacional concreto, el cambio de estrategias (bruneriano) en la categorización de objetos de acuerdo a reglas perceptuales, funcionales o nominales; $y$, para añadir un ejemplo reciente, el cambio en algunos aspectos y la continuidad en otros, del uso de la fantasía en los niños versus en los adultos (Woolley, 1997). 
La psicología del desarrollo se caracteriza por su objeto formal de continuidad/estabilidad y por el cambio en el curso del tiempo. No posee un objeto material independiente. Damon (1997, p. 74) afirma: "Podemos formular preguntas sobre el desarrollo de cualquier actividad humana -inclusive acerca de la tarea de hacer preguntas sobre el desarrollo". No se caracteriza por un grupo de edad o una población determinada, p.e. niños, mayores o retardados mentales. La continuidad/cambio conductual se aplica a todas las edades y poblaciones.

Existen razones históricas para la separación de estas disciplinas. Tanto la psicología experimental como la correlacional se inspiraron en la ciencia y la fisiología del siglo XIX; la psicología del desarrollo, por el contrario, se inspiró en el análisis de la historia y la evolución social del siglo XIX y en el análisis del origen de las especies de Darwin. Esta posición incluía perspectivas metateóricas y daba acceso tanto a argumentos empíricos como no empíricos.

El pensamiento histórico del siglo XIX sostenía la creencia del progreso científico y social. Comte distinguía peldaños, que formaban una escalera desde la especulación teológica hasta el conocimiento científico. Más tarde, la preocupación de Piaget era la génesis de la humanidad desde una criatura biológica a una lógica. El consideró seriamente conducir un estudio filosófico-histórico pero prefirió estudiar esta génesis en el niño, "el sitio del descubrimiento, donde todo ocurrió" (Piaget, 1970).

La idea de Darwin de la evolución biológica es propuesta como otra importante fuente del pensamiento evolutivo. Richards (1989, 1992) la relativiza. La evolución es considerada como resultado de factores aleatorios, aún cuando los productos de la evolución pueden impresionar como ordenados y siguiendo un plan. Los evolucionistas dudaron mucho en abandonar la idea de un plan que resultaba en un sendero común y aceptaron a regañadientes la idea de igualdad y diversidad en el desarrollo (Damon, 1997). La idea del seguimiento de un sendero común hacia algo mejor, superior, más equilibrado difícilmente se puede evitar (Tappan y Brown, 1992) pero puede estrechar la perspectiva de los fenómenos reales. 
Noam (1996) ilustra esto con la perspectiva cognitivo-evolutiva sobre la delincuencia. Aquí seduce asumir y hacer esfuerzos por encontrar que los niveles superiores de desarrollo moral y social no son alcanzados por los delincuentes, lo cual puede obstaculizar la búsqueda de razones más sutiles de la conducta delictiva y descuidar el hecho que los niveles superiores no impiden la delincuencia.

Es este pensamiento evolutivo histórico y biológico el que "cargó" a la psicología del desarrollo con una perspectiva metateórica relacionada con los etapas progresivas y la variabilidad aleatoria. Además, los constructos eran semánticos, divergentes, por ejemplo, a la orientación de Weber y Fechner sobre modelos matemáticos que conectan la realidad psicológica y física. Asimismo, con el fin de captar el significado de estos constructos, se empleaban métodos empíricos y no empíricos (analíticos y hermenéuticos).

Recientemente, las etapas progresivas así como la igualdad y diversidad han sido aceptadas como una "divergencia fructífera" (Bruner, 1997). Kagan, por ejemplo, dice: "La naturaleza presenta lo uniforme y lo variable en cada fenómeno, haciendo posible que los científicos escojan el ángulo que desean explorar" (1992, p. 990).

En suma, la psicología del desarrollo como "tercera disciplina" se caracteriza por su objeto formal. Esta perspectiva coloca a las teorías/ constructos en el corazón del asunto. Los antecedentes históricos le proveen tanto progresos transformacionales, como igual cantidad de cambios variacionales; así como métodos analíticos y hermenéuticos, aparte de métodos de investigación empíricos. En la búsqueda del significado de continuidad/cambio conductual, la psicología del desarrollo adopta fácilmente nuevos constructos y modelos, por ejemplo, la teoría de sistemas dinámicos, los enfoques de lapso de vida y curso de vida, los modelos neurales, los modelos de interacciones persona-contexto y ecológicos. 


\section{Evaluación psicológica: cuatro componentes y tres niveles}

Galton es el padre de la evaluación psicológica. El separó el estudio de la inteligencia (1883) y la personalidad (1884) de la hegemonía de la filosofía. Se le ofrecían a los sujetos tareas y preguntas y sus desempeños eran juzgados y computados. Esto resultaba en la medición de las diferencias individuales en inteligencia y personalidad.

El significado de evaluación rápidamente se amplió cuando fue utilizada en la selección, colocación y ayuda de las personas. Walsh y Betz (1990), por ejemplo, describen la evaluación como el proceso de ayudar a las personas a enfrentar sus problemas, recolectando, comprendiendo, integrando información y conduciendo intervenciones. De Bruyn (1992) la considera como un proceso de decisión sistemático acerca de qué información recolectar y qué acciones tomar para un cliente. Jager y Petermann (1992, p. 11) apuntan a la información que es recolectada y analizada para la intervención. Describen qué se puede evaluar: las características de la(s) persona(s), grupos, instituciones, situaciones e inclusive, objetos.

Tanto De Bruyn (1992) como Jager et al. (1992) enfatizan las prescripciones científicas para el proceso diagnóstico. Ellos toman la prueba de hipótesis procedente de la investigación analítico-empírica (experimental y correlacional) como un modelo prescriptivo. Esto otorga respetabilidad científica a la evaluación.

Con el fin de describir la evaluación en una forma comprensiva se propone una matriz de cuatro componentes por tres niveles, la cual sirve a propósitos heurísticos y no agrega nada nuevo a la evaluación. Los componentes son los siguientes: teoría de los tests, constructos psicológicos, tests y cuestionarios y proceso diagnóstico. Los mismos están involucrados en toda actividad evaluativa. Los componentes linguísticos Gramática, Semántica, Fonología y Pragmática sirven como analogía. Al igual que la evaluación, el lenguaje puede describirse con diferentes componentes. No existe teoría que cubra todos los componentes y no es necesario que lo haga (Bloom, 1993, p. 40). Aunque cada componente 
es indispensable para hablar, escuchar y comunicarse, pueden abordarse en forma separada e investigarse independientemente. Lo mismo se aplica a los cuatro componentes de la evaluación.

1. La teoría clásica de los tests (TCT) contiene estimados de los errores en las observaciones (confrabilidad) y la teoría de respuestas a los ítemes (TRI) ofrece modelos para explicar la probabilidad de una respuesta a un ítem (ver, por ejemplo, Goldstein y Wood, 1989). Esto es análogo a la teoría de la sintaxis, por ejemplo, la de la gramática transformacional.

2. Las teorías incluyen redes de constructos que se refieren a las diferencias entre las personas. No hay razón para excluir las teorías del desarrollo conductual. La evaluación siempre es acerca de contenidos, esto es, acerca de las conductas, cogniciones y emociones de los sujetos. Esto es análogo a la semántica, por ejemplo, los estudios para determinar las dimensiones del espacio semántico.

3. Los procedimientos de los tests, cuestionarios y observaciones incluyen la unidad más pequeña de evaluación: el ítem, la pregunta, la tarea, la categoría conductual. Esto es análogo a la fonología, que describe las unidades más pequeñas (fonemas) e investiga la combinación de reglas para poder formar palabras con significado. El ítem tiene que cumplir con requisitos psicométricos y relacionados a los contenidos para que pueda contribuir válidamente a la medición a través de una evaluación de un constructo.

4. El proceso diagnóstico es una actividad con un inicio (una pregunta, un problema), un curso (análisis científico del problema, medición de conductas, prueba de hipótesis) y una meta (un diagnóstico, la solución de un problema, un consejo). Es análogo al estudio de la pragmática del lenguaje, al distinguir las reglas que permiten comunicar el mensaje adecuadamente y seguir los pasos necesarios y apropiados para responder a la pregunta diagnóstica.

Se distinguen tres niveles. Más obvio es el segundo, que es el nivel "intermedio" de las teorías/constructos psicológicos (ver, por ejemplo, Snow, 1973 para la distinción de los niveles). La actividad de evaluación 
se apoya en la operacionalización de las conductas que se supone están involucradas en el constructo. Sin embargo, las personas legas tienen sus propios constructos. Esto constituye el primer nivel. Pueden estudiarse por derecho propio y algunos psicólogos los consideran relevantes para los constructos de rasgos psicométricos de inteligencia y personalidad, que constituyen el nivel "intermedio" (ver, respectivamente, Sternberg, 1985; Hofstee, 1994). El tercer nivel se refiere a la metodología modelo o al método hipotético-deductivo. La primera contiene al mismo tiempo una teoría y una medición de algunos elementos básicos y de la estructura de la conducta. Modelos para las respuestas a los ítemes de los tests son proporcionados por cerca de 50 modelos TRI (Goldstein y Wood, 1989). La teoría de sistemas dinámicos, por ejemplo, está siendo utilizada porque parece prometedera para la evaluación de temas de continuidad/cambio específicos; digamos, las transiciones de etapas o el crecimiento (ver, respectivamente, Van der Maas, 1993; Van Geert, 1994).

Estos niveles existen tanto en la investigación como en la evaluación porque uno puede seguir un procedimiento de abajo-arriba o de arribaabajo. En la evaluación uno puede, por un lado, empezar con un contenido conductual de sentido común definido a la ligera que es escudriñado y analizado empíricamente con técnicas exploratorias para definir su dimensión conductual. De otro lado, uno puede empezar con un modelo vacío cuyos elementos y relaciones están estrictamente definidos. Los datos deben adecuarse al modelo; de lo contrario, el modelo no se aplica y debe cambiarse. A veces, sin embargo, no es cambiado y se echa la culpa a los datos, por ejemplo, por la limitada confiabilidad de las observaciones o la ambigüedad del constructo. La mayor parte de los constructos psicológicos están en un nivel intermedio, es decir, en algún lugar entre estos dos niveles, porque los constructos no son ni borrosos conceptos de sentido común ni modelados formalmente o investigados de manera hipotético-deductiva.

La pugna entre los niveles atrae mucha atención. El sentido común es considerado tanto un reto como un freno para el progreso científico. Los constructos psicológicos pueden describirse tanto como una ayuda en comparación a las creencias populares como inexpresivos o triviales. 28 
Finalmente, los especialistas en TRI pueden rotular ambos niveles como no científicos porque carecen de modelos de medición (Fischer, 1991).

Esta pugna puede ponerse en perspectiva. Todos tienen la meta de captar el significado de las conductas, cogniciones, emociones de las personas. Operan, sin embargo, en diferentes contextos y tienen diferentes funciones. No pueden separarse y deben confrontarse continuamente; por ejemplo, el psicólogo encargado de la selección de personal tiene que comunicar sus hallazgos científicos al empleador, quien maneja teorías populares, y el diseñador de modelos TRI tiene que surtir a su modelo de una interpretación para que pueda ser comprendido por el psicólogo.

En suma, la evaluación empezó como la medición de diferencias individuales. La inteligencia y la personalidad dejaron de ser temas de análisis filosóficos. Rápidamente se expandió y la evaluación de los clientes llegó a compararse con el proceso de efectuar una investigación empírica. En este artículo, la evaluación se describe comprensivamente con cuatro componentes y tres niveles (Cuadro 1). Las teorías/constructos psicológicos están tanto en la fila como en la columna. No son lo mismo porque representan un nivel y un componente, respectivamente.

\section{Evaluación del desarrollo}

El segundo nivel, intermedio, de los constructos evolutivos es más elaborado. Es el corazón del asunto porque contiene una perspectiva sobre las preguntas permanentes de continuidad/estabilidad y cambio conductuales. Este nivel se confronta particularmente con los cuatro componentes de la evaluación. Se agregan algunos ejemplos de las contribuciones del primer y el tercer niveles a estas preguntas evolutivas.

\section{Teoria de los tests y constructos evolutivos}

La confiabilidad (error de medición) es el concepto central de las TTC. Para estimar la confiabilidad, se computan los coeficientes de estabilidad y consistencia interna. Si el constructo evolutivo implica estabilidad en el 


\section{Cuadro 1}

\section{Descripción Comprensiva de la Evaluación a Través de Componentes y Niveles}

\begin{tabular}{|c|c|c|c|c|}
\hline \multirow[t]{3}{*}{$\begin{array}{l}\text { Niveles de la } \\
\text { evaluadon }\end{array}$} & \multicolumn{4}{|c|}{ Componentes de la evalinación } \\
\hline & Teoría de los tests & $\begin{array}{l}\text { Teorías/construc- } \\
\text { tos psicológicos }\end{array}$ & $\begin{array}{l}\text { Tests, instrumen- } \\
\text { tos, procedimien- } \\
\text { tos observacionales }\end{array}$ & $\begin{array}{l}\text { Proceso de evalua- } \\
\text { ción }\end{array}$ \\
\hline & Componente 1 & Componente 2 & Componente 3 & Componente 4 \\
\hline $\begin{array}{l}\text { Creencias acerca } \\
\text { del desarrollo im- } \\
\text { plícitas/de sentido } \\
\text { común } \\
\text { Nivel } 1\end{array}$ & $\begin{array}{l}\text { v. g. } \\
\text { Ideas de } \\
\text { confiabilidad, } \\
\text { consistencia } \\
\text { derivadas del } \\
\text { sentido común }\end{array}$ & $\begin{array}{l}\text { v. g. } \\
\text { Creencias acerca } \\
\text { de estabilidad o } \\
\text { cambio en la con- } \\
\text { ducta }\end{array}$ & $\begin{array}{l}\text { v. g. } \\
\text { Uso de etapas, se- } \\
\text { gún el sentido co- } \\
\text { mún. Por ejemplo, } \\
\text { estar en la fase obs- } \\
\text { tructiva }\end{array}$ & $\begin{array}{l}\text { v. g. } \\
\text { Jugar y predecir la } \\
\text { conducta futura de } \\
\text { acuerdo al sentido } \\
\text { común }\end{array}$ \\
\hline $\begin{array}{l}\text { Teorías/construc- } \\
\text { os evolutivos } \\
\text { Nivel } 2 \\
\text { intermedio }\end{array}$ & $\begin{array}{l}\text { v. g. } \\
\text { Estabilidad en el } \\
\text { curso del tiempo, } \\
\text { consistencia inter- } \\
\text { na }\end{array}$ & $\begin{array}{l}\text { v. g. } \\
\text { Constructos evolu- } \\
\text { tivos etápicos y no } \\
\text { etápicos }\end{array}$ & $\begin{array}{l}\text { v. g. } \\
\text { Tests infantiles y } \\
\text { para bebés }\end{array}$ & $\begin{array}{l}\text { v. g. } \\
\text { Uso de constructos } \\
\text { evolutivos para el } \\
\text { diagnóstico, consejo } \\
\text { e intervención }\end{array}$ \\
\hline $\begin{array}{l}\text { Modelo matemá- } \\
\text { tico del desarrollo } \\
\text { Nivel } 3\end{array}$ & $\begin{array}{l}\text { v. g. } \\
\text { Modelos TRI para } \\
\text { el desarrollo de } \\
\text { rasgos latentes }\end{array}$ & $\begin{array}{l}\text { v. g. } \\
\text { Modelo de creci- } \\
\text { miento y de tran- } \\
\text { siciones en las eta- } \\
\text { pas }\end{array}$ & $\begin{array}{l}\text { v. g. } \\
\text { Modelos de esca- } \\
\text { las ordinales para } \\
\text { el desarrollo }\end{array}$ & $\begin{array}{l}\text { v. g. } \\
\text { Modelos de decisión } \\
\text { normativos }\end{array}$ \\
\hline
\end{tabular}

curso del tiempo a nivel de una muestra de personas, el coeficiente es relevante, Informa que el orden de ubicación de las personas en la misma conducta no ha cambiado. El coeficiente no es relevante si uno espera cambios en la conducta en el curso del tiempo y/o estabilidad diferencial de grupos especificos o personas individuales.

Si el constructo evolutivo implica que un conjunto de conductas medidas en un tiempo determinado en una población determinada es completamente intercambiable, el coeficiente de consistencia interna es 
relevante. No es apropiado si los ítemes representan niveles evolutivos, es decir, si aparecen (o desaparecen) diferentes conductas en el curso del tiempo en los individuos y en los grupos.

Algunas veces la diferencia entre los puntajes obtenidos por un grupo en dos ocasiones es interpretada como desarrollo. Las TTC contienen estimados de la confiabilidad de diferentes puntajes. El nivel de este coeficiente depende de las confiabilidades de ambas medidas y de la falta de correlación entre las dos. Será más alto si la primera medida no predice el resultado de la segunda. La relevancia de este coeficiente depende del constructo de continuidad/cambio específico.

Estos coeficientes contienen solamente información indirecta acerca del desarrollo y están sesgados hacia la estabilidad. Levy (1974, p. 21) arguye inclusive: “...las teoría de los tests contienen una buena pragmática pero su relación con cualquier teoría psicológica es cuestionable". Van Geert (1994) critica la interpretación de la variabilidad como un error porque la variabilidad es necesaria para el desarrollo. Estas críticas apuntan al hecho que debe haber un vínculo conceptual entre el contructo evolutivo y los coeficientes que estiman el error en las observaciones. Finalmente, los estimados de confiabilidad no pueden interpretarse en un nivel individual. De modo que no hay espacio para una pautación evolutiva individual.

La continuidad y el cambio pueden analizarse con TRI. Pueden considerarse como un modelamiento de tercer nivel, de una conducta pequeña (una respuesta a un ítem) con una teoría pequeña (un rasgo latente). Los cambios reales en los rasgos latentes pueden distinguirse de los accidentales (Fischer, 1991). Estos modelos no están involucrados frecuentemente en las preguntas de cambio/estabilidad. Si se aplican, la razón para ello es casi siempre para mostrar las posibilidades del modelo y no para obtener conocimientos acerca de la continuidad/cambio. Según Goldstein y Wood (1989), las TRI se describen mejor como Modelos de Respuesta a los Itemes (MRI). Estos autores enfatizan la relación de los contenidos, esto es, los constructos conductuales y la teoría de los tests. 
En suma, las teorías de los tests no pueden aplicarse rutinariamente a las preguntas de continuidad/cambio porque no contienen información directa sobre el tema. Las TCT son fundamentalmente modelos de errores y los modelos TRI suelen desarrollarse independientemente de las preguntas evolutivas.

\section{Las teorias psicológicas y los constructos evolutivos especificos}

La mayor parte de los constructos en la disciplina experimental y correlacional no son conceptos de sentido común ni están modelados formalmente. Como constructos de nivel intermedio contienen elementos semánticos y relaciones entre estos elementos. Se utilizan para operacionalizar el constructo. Los constructos contienen indicaciones y restricciones pero no prescripciones exactas sobre qué y cómo operacionalizar.

Sin embargo, una vez realizada la operacionalización, contribuyen a la clarificación del constructo. Meehl (1992) considera este procedimiento "Munchhausen" como fructifero y rechaza el requisito de que un constructo esté completamente definido desde el principio. Los constructos semánticos y la investigación empírica apropiada se acompañan entre sí en la captación del significado del constructo, como Cronbach \& Meehl (1955), Campbell \& Fiske (1959) y Messick (1989) han sostenido convincentemente.

Los constructos evolutivos también son predominantemente de nivel intermedio. Además, implican la idea de organización conductual en el curso del tiempo. No se puede excluir por adelantado ningún patrón, ni siquiera uno aleatorio. Los constructos contienen conjeturas acerca de las relaciones internas de (clases de) conductas (p.e. "etápicas o "no etápicas") y de las relaciones con otros constructos (p.e. pautación de etapas o una variedad de senderos evolutivos). Asimismo, los constructos evolutivos representan las ideas históricas de progreso, por un lado, y de variabilidad aleatoria por el otro. Se utilizan aquí la investigación empírica y los procedimientos analíticos y hermenéuticos. 
Los constructos de etapa y no-etapa del desarrollo ilustran estas características. El constructo de etapa no es ni formal ni de sentido común sino de nivel intermedio. Se acompaña tanto de argumentos empíricos como no empíricos.

"Etapa" implica que diferentes conductas son las mismas de una u otra manera. Brainerd (1978) interpretó esto como que están correlacionadas. Él no encontró tales correlacionnes y concluyó que la etapa de operaciones concretas no existía. Piaget definió esta etapa estructuralmente. Los "decalages" que encontró realmente fueron desalentadores. De modo que la investigación empírica no era simplemente una ilustración no comprometida de la estructura. De otro lado, los decalages no lo llevaron a abandonar la idea estructural. El concepto es un híbrido de procedimientos empíricos y analíticos. Kohlberg trató de encontrar soporte empírico para las seis etapas de desarrollo moral y distinguió inclusive una etapa $5 \mathrm{~B}$ que explicaba las respuestas de los estudiantes que aparentemente eran de la etapa 2 . El manual ampliado (Kohlberg y Colby, 1987) contiene procedimientos hermenéuticos, esto es, diferentes respuestas pertenecientes a una etapa debido a un significado común más profundo.

Las etapas implican transiciones. Las personas permanecen un tiempo en una etapa y luego se trasladan a otra etapa. ¿Cómo se puede evaluar si ocurre una transformación? Para responder esta pregunta, se puede seguir la misma discusión que se hizo con el concepto de etapa. ¿Se puede preguntar si existe una transformación empírica, estructural o significativa?

Las etapas deben ordenarse en una secuencia. ¿Cómo se puede conceptualizar un patrón así? En primer lugar, se puede señalar que es un patrón que uno simplemente puede observar. Se asume que existe un ordenamiento natural en las formas de razonar y de comportarse socialmente en el curso del tiempo. En segundo lugar, se puede señalar que es un patrón taxonómico, una jerarquía lógica, en la que una etapa $n$ incluye una etapa $n-1$ (ver, por ejemplo, Brandtstdter, 1990). Si las conexiones no son empíricas sino analítico/lógicas, la investigación empírica parece superflua. Flavell (1971) consideró que la investigación empírica sobre tal 
tópico (el estudio de Kofsky, 1966, sobre los niveles evolutivos de los conceptos bajo su supervisión) es un tipo benigno de error científico. En tercer lugar, se puede argüir que el sistema cognitivo, conductual, motivacional y social cambia en sí mismo. El sistema se reorganiza por sí mismo en un intercambio con el ambiente natural, social, simbólico, cultural.

Las teorías socio-culturales no describen etapas estructurales progresivas sino que definen las condiciones bajo las cuales puede ocurrir el desarrollo. El desarrollo es un cambio en la cognición, conducta, emoción del sujeto y de la posición en su mundo social y cultural. El desarrollo está situado ,en una parte sustancial, en el ambiente social y cultural (Heymans, 1994). Una persona (niño, adulto) hace evidente a una audiencia específica (padres, profesores, amigos, colegas, representantes de instituciones) de que puede ejecutar ciertas acciones (leer, controlar esfínteres, mantener relaciones entre pares, ser ejecutivo). La audiencia llega a persuadirse (o no) y la ersona se siente orgullosa (o avergonzada) Se le adscribe una nueva identidad a la persona porque tiene una nueva competencia a su disposición y es tratada por la audiencia de ahora en adelante como que posee la competencia.

El contexto elicita desarrollo si hay una "tarea evolutiva"; que puede ser cumplida o no; que sea juzgada por la persona o institución involucradas; para la cual la persona es responsable; el fallar tiene consecuencias para el estatus moral de la persona; en las tareas, el "desarrollador" y el que "va a desarrollarse" están involucrados y la tarea conecta a la persona con el ambiente socio-cultural. La persona jamás aprende algo porque sí; lo que aprende está enclavado y es valioso en el ambiente socio-cultural.

La perspectiva contextualizada socio-culturalmente posibilita muchos senderos evolutivos. Al final, uno puede definir el desarrollo como un "proceso estocástico multideterminado, en parte único al individuo; una característica que se refleja en marcadas interacciones y en la no-linealidad de los datos entre los individuos" (Bergman, 1996, p. 1). 
Los constructos evolutivos de nivel intermedio no son formales, esto es, contienen ambigüedad. Los modelos matemáticos de nivel 3 ingresan al nivel intermedio en dos formas. En primer lugar, a veces se las recomienda para evitar ambigüedades (semánticas) (ver, por ejemplo, Roskam, 1991). Esto, sin embargo, no resuelve el problema de ambigüedad porque el modelo que se adecúa debe ser interpretado y comunicado, de modo que se tiene que usar elaboraciones semánticas. En segundo lugar, se puede modelar el desarrollo de una conducta específica. Sin embargo, generalmente ocurre al revés. Existe un modelo que promete, que se extrae de una ciencia diferente y que se usa para los constructos evolutivos. Se elaboran aquí dos ejemplos.

Van Geert (1991) desarrolló un modelo de sistema dinámico para el crecimiento cognitivo y lingüístico. Es intuitivamente atractivo considerar el crecimiento como un sistema dinámico. Algo puede crecer pero existen limitaciones. Simultáneamente al crecimiento parece desarrollarse una restricción para crecer más. Van Geert propone un modelo de crecimiento logístico con retroalimentación demorada. Lo toma prestado de la biología de poblaciones:

$$
\mathrm{Lt}+1=\mathrm{Lt}(1+\mathrm{r}-\mathrm{r} \cdot \mathrm{Lt} / \mathrm{K})
$$

Esta fórmula describe cómo el crecimiento a un tiempo $t+1$ es causado por el crecimiento en el tiempo $t$ y por dos parámetros adicionales $\mathrm{r}$ y K. R se refiere a la tasa de crecimiento y $\mathrm{K}$ a la capacidad de crecimiento o de arrastre. La capacidad utilizada es la razón entre el tamaño de la población corriente y la densidad de equilibrio, que es el tamaño de la capacidad de arrastre del ambiente. De modo que existe una capacidad utilizada para el crecimiento: $\mathrm{Lt} / \mathrm{K}$ y existe todavía una capacidad no utilizada: $(\mathrm{K}-\mathrm{L} \mathrm{t}) / \mathrm{K}$.

La teoría del caos es parte de la teoría de los sistemas dinámicos. Parecía apropiado para describir y explicar las transiciones de etapas. Van der Maas (1993) la utilizó para la descripción y explicación de la transición del pensamiento preoperacional al operacional concreto. Si una transición es una 
catástrofe "real”, deben presentarse ocho "banderas": Un salto rápido; multi o bimodalidad de las respuestas; variación y oscilación elevadas en las respuestas; divergencia respecto de la respuesta lineal; demora en la reinstalación después de un disturbio; inaccesibilidad, no es posible ir hacia otra distribución; histeresis (este fenómeno aparece cuando para un solo y mismo valor de la variable independiente, están presentes más valores de la variable dependiente; no hay un punto único sino un área, un rango de la variable independiente en donde ocurren el cambio, la transición o la reversión); divergencia de la respuesta.

En la transición del pensamiento preoperacional al operacional concreto, se encontraron distribuciones bimodales de las respuestas así como elevadas variaciones entre ellas (Van der Maas, 1993, p. 11-24).

Este modelo de crecimiento y de transición de etapas es tomado de la biología matemática y de las matemáticas y contribuye a la captación del significado de continuidad/cambio, de transformación. Al momento actual, estos modelos generan muchas expectativas. Barton (1994) advierte sobre su abuso y lo considera solamente como una metáfora. Bogartz (1994, p. 314) arguye que el modelo puede complicarse fácilmente porque en principio cualquier acontecimiento anterior tiene un efecto sobre el presente. Esto crece exponencialmente de modo que los resultados no pueden computarse.

Las creencias de continuidad/cambio derivadas del sentido común, pertenecientes al Nivel 1, se estudian por derecho propio y muestran diversidad. Un ejemplo es un estudio de Ross (1989) en el que 100 sujetos, de 5 a 85 años de edad, dibujaron diagramas para 83 atributos conductuales. El $74 \%$ de los diagramas encajaba con uno de los cuatro prototipos: (a) estabilidad, una línea recta $(21,9 \%)$; (b) una curva en forma de $U(13,6 \%)$; (c) una curva en forma de $U$ invertida $(20,6 \%)$ y (d) un rápido incremento en los primeros años seguido por estabilidad (18,1\%). No se extrajeron patrones aleatorios. Estas creencias tuvieron un efecto sobre las conductas, por ejemplo, si una característica era percibida como estable, se subestimaban los cambios reales. 
Gergen, Gloger-Tippelt y Berkowitz (1990) investigaron, por ejemplo, las creencias de mujeres americanas y alemanas acerca del desarrollo de los bebés, infantes y niños en edad de caminar. Los dos grupos apoyaron la teoría "organísmica", compartiendo la creencia de un desarrollo ordenado entre los 0 y 50 meses. Además, relacionaban ciertas actividades educacionales/pedagógicas con diferentes edades. En el primer año, éstas se dirigían a las emociones (comodidad, calma) y más adelante, a la interacción social (jugar, elogiar y exigir) y la cognición (explicar, solucionar problemas y estimular la fantasía).

En suma, los constructos de nivel intermedio son el corazón del asunto. Como constructos semánticos son ambiguos y no pueden estar completamente determinados desde el principio. Además, contienen ideas no meta-teóricas, por ejemplo, de progreso o de variabilidad aleatoria. La investigación empírica, el análisis no empírico (analítico y hermenéutico) y la conciencia de sesgos meta-teóricos contribuyen a la captación del significado de continuidad/cambio conductual. Los conceptos de etapa piagetiana y de no etapa vigotskiana alertan sobre los enigmas del desarrollo (Bruner, 1997). Los modelos matemáticos tienen que ver, entre otros aspectos, con la discusión sobre el crecimiento y la transición de etapas y añaden significado a los fenómenos evolutivos específicos. Las creencias de sentido común son estudiadas por derecho propio y'son diversas. Operan en forma relativamente independiente de los constructos de nivel intermedio.

Tests, cuestionarios, procedimientos observacionales y constructos evolutivos

En las revisiones de los tests, los tests evolutivos se refieren a los tests para bebés e infantes, por ejemplo, las Escalas de Desarrollo Infantil de Bayley y las Escalas de Desarrollo de Denver. Su meta es evaluar las diferencias individuales en los desempeños mentales y motores.

Pocos tests han sido elaborados con un constructo evolutivo en mente. Kerssies, Rensen, Oppenheimer y Molenaar (1989) han elaborado escalas ordinales de desarrollo (Uzgiris y Hunt, 1975). Se basan en los seis estadíos 
de la etapa sensoriomotor de Piaget, quien representó estos estadíos, a diferencia de los posteriores, no como una estructura lógico/matemática. Los formuló teniendo en mente el equipo biológico del niño y utilizando las observaciones de los juegos con sus hijos. Kerssies et al. emplearon estas descripciones para evaluar el nivel evolutivo de niños de 0 a 2 años de edad y de niños severamente retardados en siete dominios. Cada dominio es ordenado de acuerdo a los seis estadíos, ningún estadío puede saltarse y forman una secuencia invariante.

Las escalas contienen entre 7 y 14 ítemes y se refieren a "seguimiento visual de objetos y permanencia del objeto; uso de medios para conseguir acontecimientos deseados; imitación vocal; imitación gestual; causalidad operacional; construcción de relaciones objetales en el espacio; esquemas para la conexión de objetos". Los ítemes son puntuados en 6 niveles. La $\mathrm{H}$ de Loevinger (1957) era muy alta (.90-1.00). Esta $\mathrm{H}$ tenía que ser usada porque los estimados de consistencia interna son inapropiados. Los autores reportan altas correlaciones de las seis escalas y la edad (.84 -.96). Estas son probablemente algunas de las muy pocas correlaciones altas entre edad y conducta, que se han encontrado.

Aún cuando desde una perspectiva teórica evolutiva se trata de un instrumento interesante, su utilidad es modesta porque la edad predice casi perfectamente el nivel evolutivo en niños normales y la aplicación del test consume tiempo y es compleja.

Los siete dominios correlacionaron alto tanto en sujetos normales (.76 -.95) como en impedidos (.65 -.91). De modo que desde el punto de vista del análisis factorial hay una sola dimensión: el desarrollo sensoriomotor. Finalmente, Rensen (1994) computó las correlaciones entre los puntajes de esta Escala y los puntajes de la Escala de Bayley. Ella encontró correlaciones sustanciales.

Otro ejemplo es el estudio de Verweij (1994) sobre el carácter evolutivo de la transitividad. El utilizó tareas de transitividad de números, sustancias y longitudes $(A>B ; B>C-(A>C)$ y las ajustó a diversos modelos. Un 
modelo ordinal (Mokken, 1971) se ajustó bien a los datos. Además, el autor correlacionó los puntajes de la escala con los desempeños en tests de aritmética y lectura y encontró correlaciones sustanciales. En este estudio, hay un constructo de continuidad/cambio que se vincula con métodos escalares.

En suma, la elaboración de tests bajo la orientación de constructos de continuidad/cambio es poco frecuente y está limitada a algunas etapas piagetanas. No están presente los constructos no-etápicos. Es posible agregar aquí los estudios longitudinales de algunos constructos evolutivos, por ejemplo, control del ego y resiliencia del ego. Los resultados empíricos muestran que hay más de un solo sendero evolutivo (Ozer y Gjerde, 1989).

\section{El proceso diagnóstico y los constructos evolutivos}

Este proceso está diseñado principalmente de acuerdo a las reglas de la investigación experimental y correlacional: el ciclo empírico y las hipótesis que prueban los modelos diagnósticos (De Bruyn, 1992). Las consideraciones evolutivas entran a tallar en el proceso, por ejemplo, seleccionando los constructos y los tests/instrumentos. Además, los efectos de la intervención pueden definirse considerando el conocimiento de los cursos evolutivos. En la práctica, esto es limitado. La confianza en tests de validez predictiva implica la convicción de que las diferencias existentes entre las personas pueden reproducirse en un punto temporal más tardío.

Los modelos matemáticos de nivel 3 influyen en elementos específicos del proceso, por ejemplo, para describir y modelar cómo el evaluador integra los resultados de los tests y los datos observacionales; como en el caso de los modelos lineales para la integración de información. El proceso de evaluación puede ser concebido como una decisión entre alternativas. Esto posibilita el uso de modelos de decisión normativos, por ejemplo, las reglas bayesianas y los modelos de utilidad de multi-atributos. Estos modelos no incluyen tópicos de continuidad/cambio.

La influencia de las creencias del nivel 1 en el proceso de evaluación apenas es reportada. Posiblemente creencias implícitas operan en acotacio- 
nes como "el problema desaparecerá; el problema puede manejarse en un momento apropiado del futuro; el problema debe ( $y$ puede) abordarse inmediatamente.".

En suma, el proceso diagnóstico está dominado por las disciplinas experimentales y observacionales. El proceso puede enriquecerse implementando constructos evolutivos apropiados de nivel 2. El estudio de las creencias de nivel 1 es interesante en sí mismo y posiblemente tiene efecto sobre el diagnóstico y las acciones resultantes (Ross, 1989). Los modelos matemáticos de nivel 3 se refieren a la integración de información hecha por el evaluador y a la optimización de decisiones. No incluyen preguntas de continuidad/cambio.

\section{Resumen y conclusiones}

La psicología evolutiva puede concebirse como la tercera disciplina de la psicología científica. La idea de Cronbach de convertirla en parte de una disciplina observacional no es fructífera. Carece del énfasis sobre la continuidad/cambio conductual. Los constructos evolutivos son constructos de nivel "intermedio", esto es, ni de sentido común ni formales. Esto implica que los constructos son semánticos y ambiguos. Describen tanto el cambio variacional como el transformacional. Se utilizan diferentes fuentes para obtener conocimientos sobre el tema de continuidad/cambio: ideas históricas metateóricas, mútodos analíticos y hermenéuticos y por último aunque no por eso menos importante, la investigación empírica. Todas ellas pueden contribuir al conocimiento del desarrollo conductual. A veces se le acusa a la psicología evolutiva de no ser una ciencia completamente empírica. Esto es verdad pero inevitable y resulta ingenuo tratar de esquivar el tema o de comportarse como si existiera una ciencia completamente empírica, como Meehl (1992) lo demuestra palmariamente. Este énfasis en el nivel intermedio no desconoce las contribuciones del primer y tercer niveles. Este juego de obtener significados se parece a los análisis de Cronbach y Meehl (1995); Campbell y Fiske (1959) y Messick (1989) de validez de constructo. 
Toda pregunta de evaluación debe enfrentar más o menos directamente cuatro componentes: teoría de los tests (modelos para evaluar confiable y válidamente la conducta), contenido psicológico (teorías y constructos y sus relaciones), tests, instrumentos, procedimientos (para evaluar concretamente la cognición, conducta y sentimiento relevantes de los sujetos) y el proceso de evaluación (el proceso de responder a la pregunta diagnóstica).

La confrontación entre Desarrollo y Evaluación parece contribuir más por su "validez discriminante" que por su "validez convergente". La conjunción de tópicos de continuidad/cambio, especialmente los conceptos de etapa y no-etapa, con los componentes de la evaluación enfatiza lo siguiente:

Los estimados de error de las TCT y los modelos de TRI no pueden usarse rutinariamente para los fenómenos evolutivos. Los constructos evolutivos contienen una estructura y un contenido semántico. Estos no están completamente, es decir, formalmente definidos desde un principio ni se derivan simplemente del sentido común. Su significado tiene que captarse a través de métodos empíricos y no-empíricos. Estos métodos no deben confundirse (confrontar la acotación de Flavell, 1971, de la "suerte benigna de error científico": confundir la prueba lógica con la empírica). Los conceptos meta-teóricos no pueden probarse empíricamente pero al ser analizados, contribuyen al conocimiento de la continuidad/cambio.

Los tests infantiles y de bebés para evaluar las diferencias individuales o el retardo no son evolutivos. Las escalas ordinales de desarrollo son teóricamente interesantes, pero poco útiles. Faltan tests que midan el desarrollo (diferencial) en diferentes dominios. También faltan tests para patrones evolutivos diferenciales. Esto está bastante más a la zaga que los tests de inteligencia y personalidad.

El proceso de evaluación está dominado por los tests de diferencias individuales y por las pruebas de hipótesis de la disciplina experimental, así como por el interés en la validez predictiva de la disciplina correlacional. Los constructos evolutivos no entran a tallar fácilmente. La evaluación o predicción clínica de nivel 1 se compara a menudo con la predicción 
estadística. Si bien no forma parte del proceso de evaluación , en realidad tiene que usarse si, por ejemplo, los datos estadísticos no se aplican al grupo o simplemente están ausentes. El modelamiento matemático de nivel 3 es utilizado para describir al evaluador y optimizar las decisiones, no contiene consideraciones evolutivas.

Las creencias de nivel 1 acerca del desarrollo ameritan ser estudiadas por derecho propio y llevan a consecuencias conductuales para las personas legas. No está claro si es una bendición para los constructos de nivel intermedio. Las creencias pueden en realidad entrar a tallar en el proceso evaluativo. Uno tiene que estudiarlas para por lo menos percatarse de estas creencias.

El modelamiento matemático de nivel 3 para los fenómenos evolutivos es tomado de la biología y la física. Aún cuando hay muchas acotaciones críticas, contribuye a la captación del significado de los constructos evolutivos, por ejemplo, de los modelos de crecimiento y transiciones específicas.

La confrontación entre desarrollo y evaluación enfatiza que la psicología evolutiva posee constructos etápicos y no-etápicos cuyo significado (validez de constructo) puede enriquecerse a través de procedimientos de evaluación que hagan justicia a los tópicos de continuidad/cambio.

\section{Referencias}

Barton, S. (1994). Chaos, Self-organization, and Psychology. American Psychologist, 49, 5-14.

Bergman, L.R. (1996). A pattern-oriented approach to studying individual development: snapshots and processes. Informe No 825 del Departamento de Psicología, Universidad de Stockholm.

Bloom, P. (Ed.) (1993). Language Acquisition. Core Readings. Hemel Hempstead: Harvester Wheatsheaf. 
Bogartz, R.S. (1994). The future of Dynamic Systems models in developmental psychology in the light of the past. Journal of Experimental Child Psychology, 58, 289-319.

Brainerd, C.J. (1978). The stage question in cognitive-developmental theory. The Behavioral and the Brain Sciences, 2, 173-213.

Brandtstädter, J. (1990). Development as a personal and as a cultural construction. En Gün R. Semin y K.J. Gergen (Eds.), Everyday Understanding: Social and Scientific Implications. Londres: Sage Publications.

Bruner, J. (1997) Celebrating Divergence: Piaget and Vygotsky. Human Development, 40, 63-73.

Campbell, D.T. y Fiske, D.W. (1959). Convergent and discriminant validation by the multi-trait multi-method matrix. Psychological Bulletin, 56, 81-105.

Cronbach, L.J. (1957). The two disciplines of scientific psychology. American Psychologist, 12, 671-684.

Cronbach, L.J. (1975). Beyond the two disciplines of scientific psychology. American Psychologist, 30, 116-127.

Cronbach, L.J. y Meehl, P.E. (1955). Construct validity in Psychological tests. Psychological Bulletin, 52, 281-302.

Damon, W. (1997). The Handbook back pages and ours. Human Development, 40, 74-86.

De Bruyn, E.E.J. (1992) A normative-prescriptive view on clinical psychological decision making. European Journal of Psychological Assessment, 8, 163-171.

Fischer, G.H. (1991). A new method for the assessment of treatment effects. Psychologycal Assessment, 7, 117-149.

Flavell, J.H. (1972). An analysis of cognitive developmental sequences. Genetic Psychology Monographs, 86, 279-350.

Galton, F. (1883) Inquiries into human faculty its development. Londres: MacMillan.

Galton, F. (1884). Measurement of Character. Fortnghtly Review, 36, 179185. 
Gergen, K.J., Gloger-Tippelt, G. Berkowitz, P. (1990). The cultural construction of the developing child. En Gün R. Semin y K.J. Gergen (Eds.), Everyday understanding: Social and Scientific Implications. Londres: Sage Publications.

Glass, G.V. Willson, V.L. y Gottman, J.M. (1975). Desingn and analysis of time-series experiments. Boulder Colorado: Colorado Associated University Press.

Goldstein, H. y Wood, R. (1989). Five decades of item response modelling. British Journal of Mathemathical and Statistical Psychology, 42, 139167.

Heymans, P.G. (1994). Conceptualization and Operationalization of Developmental Tasks. En J. ter Laak (Ed.), Voronovo thoughts on Developmental Tasks. Utrecht University: Department of Developmental Psychology.

Hofstee, W.K.B. (1994). Who should own the definition of personality? European Journal of Personality, 8, 149-162.

Jager, R.S. y Petermann, F. (Eds.) (1992). Psychologische Diagnostik, 2e Auflage. Munich: Psychologie Verlags Union Weinheim.

Kagan, J. (1992). Yesterday's Premises, Tomorrow's Promises. Develompental Psychology, 28, 990-997.

Kerssies, I.J., Rensen, F.S.X., Oppenheimer, L. y Molenaar, P.C.M. (1989). De ordinale schalen voor het bepalen van de psychologische ontwikkeling in de sensorimotorische periode. (Ordinal scales for the determination of psychological development in the sensorimotoric period). Lisse: Swets \& Zeitlinger.

Kofsky, E. (1966). A scalogram study of classificatory development. Child Kohlberg Development, 37, 191-204.

Levy, Ph. On the relation between test theory and psychology. En P. Klinme (Ed.), New approaches in spychological Measurement. Londres: J. Wiley .

Loevinger, J. (1957). Objective tests as instruments of psychological theory. Psychological Reports, 3, 635-694.

Meehl, P.E. (1992) Factors, taxa, traits and types, differences of degree and differences in kind. Journal of Personality, 60, 118-174. 
Messick, S. (1989). Validity. En R. Linn (Ed.), Educational Measurement. (3ra. ed.) National Council on Measurement in education. Londres: Colliet MacMillan Publishers.

Mokken, R.J. (1971). A Theory and a Procedure of Scale analysis. The Hague: Mouton.

Noam, G. (1996). High-risk youth: Transforming our understanding of human development. Human Development, 39, 1.17.

Ozer, D.J. Gjerde, P.F. (1989). Patterns of personality consistency and change from adulthood though adolescence. Journal of Personality and Social Psychology, 58, 472-486.

Piaget, J. (1970). Piaget's theory. En P.H. Mussen (Ed.), Carmichaels's Handbook of Child Psychology (Vol. 1). Nueva York: Basic Books.

Rensen, F.S.X. (1994). De organisatie van sensomotorische ontwikkelingsprocessen. Een structurele vergelijking tussen de normale en geretardeerde ontwikkeling (The organization of sensorimotoric developmental processes; A comparison between normal and retarded development). Tesis doctoral, Universidad de Amsterdam, Amsterdam.

Richards, R.J. (1987). Darwin and the Emergence of Evolutionary Theories of Mind. Chicago: The University of Chicago Press.

Richards, R.J. (1992). The meaning of Evolution. Chicago: The University of Chicago Press.

Roskam, E.E. (1991). Construct validity as explanatory theory. Paper prepared for the second European Conference on Personality Assessment, Barcelona, Sept. 23-24, 1992.

Ross, M. (1989). Relation of implicit theories to the construction on Personal Histories. Psychological Review, 96, 341-357.

Sijtsma. K. y Verweij, Ac. (1992). Mokken Scale Analysis: Theoretical Considerations and an Application to Transitivity Tasks. Applied Measurement in Education, 5, 355-373.

Snow, R.E. (1973). Theory construction for research on teaching. En R.M.W. Travers (Eds.), Second Handbook for Research on Teaching. Chicago: Rand Mc Nally.

Sternberg, R.J. (1985). Implicit theiries of intelligence, creativity and wisdom. Intelligence, 15, 257-269. 
Tappan, M.B. y Brown, L.M. (1992). Hermeneutics and developmental psychology: Toward an ethic of interpretation. En W.M. Kurtines, M. Azmitia y J.L. Gewirtz. The role of values in psychology and human development. Nueva York: Wiley.

Uzgiris, I.C. y Hunt, J. McV. (1975). Assessment in infancy. Ordinal scales of psychological development. Chicago: University of Illinois Press.

Van der Maas, H. (1993). Catastrophe analysis of stagewise cognitive development: Model, method and applications. Amsterdam: Academisch proefschrift Inivertiteit van Amsterdam.

Van Geert, P. (1991). A dynamic system model of cognitive and language growth. Psychological Review, 98, 3-53.

Van Geert, P. (1994). Dynamic systems of development, change between complexity and chaos. Nueva York: Harvester Wheatsheaf.

Verweij, A.C. (1994). Scaling transitive inference in 7-12 rear old children. (Academisch proefschrift, Amsterdam: Vrije Universiteit). Enschede: Febo Druk.

Vygotsky, L.S. (1962). Thought and Language (Editado por E. Hanfmann y G. Vakar). Cambridge: The MIT Press. (Original en 1934).

Walsh, W.B. y Betz, N.E. (1990). Tests and Assessment. Nueva Jersey: Prentice Hall.

Wohlwill, J.F. (1973). The Study of behavioral development. Nueva York: Academic Press, The Child Psychology Series.

Woolley, J.D. (1997). Thinking about fantasy: Are children fundamental different thinkers and believers from adults? Child Development, 68, 991-1011. 